\title{
Особенности процесса кристаллизации в пересыщенном растворе (на примере сахарного производства)
}

\author{
Славянский Анатолий Анатольевич \\ ФГБОУ ВО «Московский государственный университет технологий и \\ управления имени К.Г. Разумовского (Первый казачий университет)» \\ Адрес: 109004, город Москва, ул. Земляной Вал, дом 73 \\ E-mail:slavyanskiyaa@mgutm.ru
}

Семенов Евгений Владимирович ФГБОУ ВО «Московский государственный университет технологий и управления имени К.Г. Разумовского (Первый казачий университет)» Адрес: 109004, город Москва, ул. Земляной Вал, дом 73 E-mail:sem-post@mail.ru

Грибкова Вера Анатольевна ФГБОУ ВО «Московский государственный университет технологий и управления имени К.Г. Разумовского (Первый казачий университет)» Адрес: 109004, город Москва, ул. Земляной Вал, дом 73 E-mail:vera_gribkova@list.ru

Николаева Наталья Валерьевна ФГБОУ ВО «Московский государственный университет технологий и управления имени К.Г. Разумовского (Первый казачий университет)» Адрес: 109004, город Москва, ул. Земляной Вал, дом 73 E-mail:nata_nik@inbox.ru

\begin{abstract}
Процессы конденсации субстанций на затравочном материале в пересыщенном растворе широко распространены в перерабатывающих производствах АПК. Так, данный процесс, трактуемый как явление кристаллизации сахарозы в вакуум-аппарате, является узловым в технологическом потоке получения кристаллического сахара на сахарном заводе. При этом подготовленный в виде тонко измельченного порошка сахарозы исходный затравочный продукт варьируется по форме и размеру, концентрации в объеме вакуум-аппарата и др. Однако теоретическое обоснование явления конденсации твердой фазы на частицах затравочного материала, как центров кристаллизации, с учетом данных факторов разработано недостаточно. В статье с целью обоснования физико-математической модели задачи в качестве окружающего частицу сахарозы объема раствора используется шаровой слой с центром в моделирующим частицу сахарозы шаром. При анализе задачи исходят из результатов ассоциированной с теорией диффузионного переноса вещества проблемы теплопереноса в шаровом слое. Обосновывается алгоритм количественного исследования зависимости концентрации сахарозы от параметров задачи и периода обработки продукта. В качестве средства количественного анализа проблемы использовался численный эксперимент. Данный эксперимент выявил адекватность результатов теоретического анализа физическому смыслу реального процесса. Получены важные в теоретическом и практическом отношении зависимости по оценке массы кристаллизованной сахарозы и времени обессахаривания раствора.
\end{abstract}

Ключевые слова: раствор сахарозы, процесс массопереноса,диффузия, период, кристаллизация, пересыщение, межкристальный раствор 


\section{Введение}

Процессы кристаллизации твердой фазы целевой субстанции (ЦС) в пересыщенных растворах продуктов растительного происхождения широко распространены в технологиях перерабатывающих производств АПК. В области процессов химической технологииупоминаются рядтеорий, объясняющих явление конденсации твердой фазы (сахарозы, соли и др.) в водном насыщенном растворе: ортокинетическая коагуляция, диффузионный перенос и др. При этом в силу своей относительной простоты, физической и экспериментальной обоснованности в теоретических вопросах, в частности, явления кристаллизации сахарозы, наибольшее распространение получила именно теория диффузионного (молекулярного) переноса массы ЦС.

\section{Литературный обзор}

Полагается, что движущей силой процесса кристаллизации служит перепад концентрации твердой фазы (молекул сахарозы) в растворе по направлению к центру кристаллизации, где концентрация твердой фазы понижена.

В результате чего в основу формализации процесса осаждения молекул сахарозы на центре кристаллизации, аналитического и количественного исследования данного явления правомерно использовать первый и второй законы Фика (Силин, 1967). Причем следует иметь в виду влияние на протекание исследуемого процесса таких показателей как температура раствора, концентрация частиц затравки, их геометрия и дисперсный состав.

Данные факторы в значительной степени влияют на параметры процессов обработки продукта.

Проблема количественного анализа процесса кристаллизациисахарозыв рабочемобъемевакуумаппарата, в том числе, по срокам обогащения межкристального раствора пересыщенным сиропом, периода времени обработки раствора в данном аппарате поднималась в работах (Валовой, 2016, с. 24-31; Виноградова, 2009, с. 1824; Гнездилова, 2005, с. 43-46; Громковский, 2008, с. 54-56; Еделев, 2016, с. 5-11; Лебедева, 2013; Михатова, 1973; Петров, 2017, с. 30-37, 2017, с. 3033, 2018, с. 38-43; Полянский, 1995; Сапронов, 1999; Семенов, 2004, с. 23-25, 2013, с. 46-50; Силин, 1967; Тужилкин, 2014, с. 66-69, 2014, с. $48-55$, 2015, с. $52-$ 58 , 2017, c. $42-48$, 2018, c. $117-121$, 2018, c. $82-85$; Фольмер, 1986; Brown, 1992, p. 35-39; Cecilia, 1984, p.21-132; Grimsey, 1994, p. 504-514; Lin, 1996, p. $25-$ 29; Mantovani, 1991, p. 23-32; Pautrat, 1996, p. 101109, p. 377-384).

В то же время вследствие своей важности для решения задачи получения сахара и других продуктов растительного происхождения высоких товарных кондиций эта проблема нуждается в дальнейшем углубленном изучении. Ниже, на примере анализа процесса кристаллизации ЦС в вакуум-аппарате, с целью обоснования параметров протекания данного процесса предлагается более обоснованное, по сравнению с известными до сих пор аналогами, его количественное моделирование.

\section{Теоретическое обоснование}

Пусть характеризуемый объемной функцией распределения $F=F(R), \mathrm{M}^{3} / \mathrm{M}^{3}$, по размеру частиц $\mathrm{R}$, м, коллектив измельченного шарообразного затравочного материала статистически равномерно распределен в некотором рабочем объеме пересыщенного водного раствора «вода + молекулы сахарозы + частицы затравочного материала» (например, в сахарном утфеле вакуумаппарата).

В качестве модели процесса массопереноса взвешенного в растворе вещества к элементам затравки выбираем изолированный (пробный) шар радиусом R, к которому из прилегающего к нему шарового слоя $R \leq r \leq \xi$ (где $r$ - радиальная координата, $2 \xi$-текущеесреднеерасстояние между центрами смежных шаров) сахарсодержащего раствора подается целевая субстанция.

При этом, если $V_{\text {ш }}$ и $V$ - соответственно, объем шаров и суммарный объем шаров и жидкости в рабочем объеме, $u=V_{m} / V$ - объемная концентрация шаров в жидкостной системе, то в соответствии с принятой геометрической моделью «жидкостная среда + система шаров» половина расстояния между двумя соседними шарами составит

$\xi=\mathrm{R}_{\mathrm{u}}{ }^{-1 / 3}, 0<\mathrm{u}<1$

Полагаем, что подвод ЦС к шарам реализуется симметричным образом, и, кроме того, с удовлетворительной точностью выполняются все, принимаемые при анализе процесса молекулярного переноса в сплошном теле, допущения по физико-механическим свойствам изучаемого объекта (Семенов, 2004, с. 23-25; 2013, c. 46-50). 
Поскольку при выбранной расчетной модели количественный анализ процесса переноса ЦС от жидкости к шарам произвольного размера весьма сложен, то в целях упрощающего допущения предполагаем, что размер шаров варьируется в некотором ограниченном интервале.

Тогда, приближенно, в основу анализа процесса массообмена между жидкостью и шарами можно выбрать шар радиусом $R=R_{\text {ср }}$, определяемый как координата центра тяжести эпюры $F=F(R)$

$$
R_{c p}=\frac{\int_{R_{1}}^{R_{2}} R F(R) d R}{\int_{R_{1}}^{R_{2}} F(R) d R},
$$

где числитель - в плоскости $(R, F(R))$ статический относительно оси $R=0$ момент плоской фигуры, ограниченной линиями $R=R_{1}, R=R_{2}$, осью $R$ и линией $F=F(R)$; знаменатель - площадь этой фигуры; $R_{1}, R_{2}$ - соответственно, минимальный и максимальный радиус.

\section{Исследование}

В качестве выражения потока $j$ концентрации от жидкости к шару (первый закон Фика) в принятой одномерной модели массопереноса в сферической системе отсчета в направлении оси $r$ принимаем

$j=D \partial c / \partial r$

где $D$ и $c$ - соответственно, коэффициент диффузии и объемная концентрация сахарозы в растворе.

Тогда в качестве исходного соотношения, описывающего кинетику распределения концентрации сахарозы в воде, выбираем, как обычно, отнесенное к сферическим координатам, с началом координат в центре пробного шара, уравнение нестационарного диффузионного массопереноса (второй закон Фика) (Семенов, 2013, c. 46-50).

$\frac{\partial \mathrm{c}}{\partial \mathrm{t}}=\mathrm{D} \frac{\partial^{2} \mathrm{c}}{\partial \mathrm{r}^{2}}+\frac{2}{r} \frac{\partial \mathrm{c}}{\partial \mathrm{r}}$

где $t$ - время.

Начальное условие по исходной концентрации, полагаемой равномерно распределенной по объему жидкостной среды (воды)

$$
c(r ; 0)=c_{n}=\text { const }(R<r<\xi),
$$

где $C_{n}$ - концентрация пересыщенного раствора.
Граничное условие на поверхности шара

$$
C(R ; t)=c_{H}(0<t<\infty),
$$

где $C_{H}$ - концентрация насыщенного раствора.

Граничное условие симметричности поля концентрации сахарозы между соседними шарами (Лыков, 1967)

$$
\frac{\partial \mathrm{c}(\xi ; t)}{\partial \mathrm{r}}=0(0<t<\infty)
$$

где расстояние $\xi$ выражается черезрадиус $R$ шара по формуле (1).

Как обычно, преобразуя граничные условия (6), (7) к однородному виду, предварительно, вводим новую переменную v, связанную с концентрациями $\boldsymbol{C}$ и $\boldsymbol{C}_{H}$ зависимостью

$$
c=v+c_{H}
$$

В результате чего краевая задача (4)-(7) относительно v принимает форму

$\frac{\partial v}{\partial \mathrm{t}}=\mathrm{D} \frac{\partial^{2} \mathrm{v}}{\partial \mathrm{r}^{2}}+\frac{2}{r} \frac{\partial \mathrm{v}}{\partial \mathrm{r}}$

$v(r, 0)=\Delta c=c_{n}-c_{H}>0(R<r<\xi)$,

$v(R, t)=0(0<t<\infty)$,

$\frac{\partial \mathrm{v}(\xi, t)}{\partial \mathrm{r}}=0(0<t<\infty)$,

где $v$ - приведенная объемная концентрация сахарозы в растворе «вода + молекулы сахарозы».

В терминах краевых задач для проблемы теплопереноса граничное условие соответствует поглощающей стенке, а условие (12) - отражающей стенке.

Решение краевой задачи (4)-(7), ассоциированное с соответствующей краевой задачей для явления теплопереноса (Лыков, 1967) и адаптированное к проблеме диффузионного массопереноса (9)-(12), имеет вид (Будак, 1956):

$v(r, t)=\sum_{n=1}^{+\infty} A_{n} \exp \left(-D \lambda_{n}^{2} t\right) \frac{\sin \left[\lambda_{n}(r-R)+v_{n}\right]}{r}$,

где

$A_{n}=2 \Delta c \frac{\left(1+\lambda_{n}^{2} R^{2}\right)\left(1+\lambda_{n}^{2} \xi^{2}\right) \int_{R}^{\xi} r \sin \left[\lambda_{n}(r-R)+v_{n}\right] d r}{\lambda_{n}^{2}(R-\xi)\left(R^{2}+\xi^{2}+R \xi+\lambda_{n}^{2} R^{2} \xi^{2}\right)}$,

где $\lambda_{n}$ - положительные корни трансцендентного уравнения 
$\operatorname{tg}\left[\lambda_{n}(\xi-R)\right]=\frac{\lambda_{n}(\xi-R)}{1+\lambda_{n}^{2} R \xi}, n=1,2, \ldots$,
$v_{n}=\operatorname{arctg}\left(\lambda_{n} R\right)$

Поток диффундирующего вещества от жидкости к поверхности шара согласно (3), (13)

$j=\frac{D d v}{d r}=D \sum_{n=1}^{+\infty} A_{n} \exp \left(-D \lambda_{n}^{2} t\right) \frac{\lambda_{n} r \cos \left[\lambda_{n}(r-R)-\sin \left[\lambda_{n}(r-R)\right]\right.}{r^{2}}$,

и поэтому объем твердой фазы, оседающей в единицу времени на единице площади поверхности частицы сахарозы в соответствии с (16) вычисляем по зависимости

$j_{r=R}=\frac{D}{R} \sum_{n=1}^{+\infty} A_{n} \lambda_{n} \exp \left(-D \lambda_{n}^{2} t\right), \mathrm{m} / \mathrm{c} . \quad$,

Количество Q сахарозы, подводимого к единице поверхности шара за время $\tau$, получим интегрируя (17) по данному временному интервалу

$Q=\int_{0}^{\tau} j(t) d t=-\sum_{n=1}^{+\infty} A_{n}\left[1-\exp \left(-D \lambda_{n}^{2} \tau\right)\right] \frac{1}{\lambda_{n} R^{\prime}} \mathrm{M}^{3} / \mathrm{M}^{2}$,

или по массе сахарозы

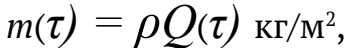

где $\rho$ - плотность сахарозы.

Поэтому масса сахарозы, конденсирующейся в $1 \mathrm{~m}^{3}$ раствора на частицах затравочного материала, составляет

$M(\tau)=m(\tau) S N, \mathrm{\kappa г}$,

где $\mathrm{m}(\tau)$ - определяется по (19); $\mathrm{S}=4 \pi \mathrm{R}^{2}$ - площадь поверхности частицы; $\mathrm{N}=\mathrm{m}_{1} / \mathrm{m}_{0}-$ количество частиц затравки в $1 \mathrm{M}^{3}$ раствора; $\mathrm{m}_{1}$ - масса вводимого затравочного материала в том же объеме, $\mathrm{m}_{0}=4 / 3 \pi \mathrm{R}^{3} \rho-$ масса частицы.

Причем, если при $\tau \longrightarrow \infty$ объем сахарозы составляет $Q_{1}$, то эффективность $\eta$ процесса конденсации твердой фазы на поверхности частицы можно оценивать на базе соотношения

$\eta=\mathrm{Q}(\tau) / \mathrm{Q}_{1}$

Откуда, на основе заданного $\eta$ может быть найдено $\tau$ как зависимость периода времени $\tau$ по относительному (к предельному) значению $\eta$ оседающего на частице массы молекул сахарозы.

Формулы (20), (21) положены в основурасчета массы сахарозы, подводимой к единице поверхности частицы затравки за время $\tau$, а также зависимость периода времени $\tau$ от эффективности $\eta$ процесса конденсации.

\section{Результаты и их обсуждение}

Предполагается, что сахарсодержащий раствор пересыщенностью $\Delta c=0,15$, при температуре $70^{\circ} \mathrm{C}$ обогащается затравкой в виде полидисперсной порошкообразной субстанции с частицами радиусами $\quad R=10^{-5} \ldots 2 \times 10^{-5} \mathrm{M}, \quad$ объемной концентрацией частиц порошка в растворе $u=2 \times 10^{-4} ; 4 \times 10^{-4}$, масса затравочного материала $m_{1}=200$ г. В соответствии с данными (Михатова, 1973) коэффициент диффузии полагали $\mathrm{D}=5 \times 10^{-10} \mathrm{M}^{2} / \mathrm{c}$.

Числовые расчеты по формулам (13), (14), (17)-(20) проводили на базе системы Mathcad, дополнительно, при определении корней уравнения (15) использовали процедуру root. Причем, принимая во внимание величину физических параметров процесса, исходя из уравнения (15), в данной статье ограничивались вычислением одного минимального по величине положительного значения корня $\lambda_{1}$ - собственного значения краевой задачи (4)-(7) (Будак, 1956). В этом случае из формул (18), (20) вытекает очевидная зависимость:

$\tau(\eta)=-\ln (1-\eta) /\left(D \lambda_{1}^{2}\right)$

Для каждого из двух зафиксированных по объемной концентрации частиц порошка в жидкости $u=2 \times 10^{-4} ; 4 \times 10^{-6} \%$ исследовались по три варианта радиуса шаров частиц данной субстанции: $R=10^{-5}, 2 \times 10^{-5} \mathrm{M}, \quad$ а $R_{\text {ср }}$ рассчитывалось по (2), при этом в качестве функции распределения принималось (по соглашению)

$F(R)=\left\{\begin{array}{c}0 \text { при } R<R_{1} \\ \frac{R-R_{1}}{R_{2}-R_{1}} \text { при } R_{1} \leq R \leq R_{2} \quad, \quad \text { при } R>R_{2} .\end{array}\right.$,

Рассчитанное при $R_{1}=10^{-5}, R_{2}=2 \times 10^{-5}$ м по (2), (22) значение $R_{\text {ср }}$ составило $1,67 \times 10^{-5} \mathrm{M}$.

Таблица первого собственного значения $\lambda_{1}$ краевой задачи для различной величины радиуса $R$ частиц затравки и их объемной концентрации $u$

\begin{tabular}{lcccccc}
\hline \multicolumn{1}{c}{$\mathbf{R}, \mathbf{M}$} & \multicolumn{2}{c}{$\mathbf{1 0}^{-5}$} & \multicolumn{1}{c}{$\mathbf{1 , 6 7 \times 1 0 ^ { - 5 }}$} & \multicolumn{2}{c}{$\mathbf{2 \times 1 0 ^ { - 5 }}$} \\
\hline$u, \mathrm{M}^{3} / \mathrm{M}^{3}$ & $2 \times 10^{-4}$ & $4 \times 10^{-6}$ & $2 \times 10^{-4}$ & $4 \times 10^{-6}$ & $2 \times 10^{-4}$ & $4 \times 10^{-6}$ \\
$\lambda_{1} \times 10^{-3}, \mathrm{M}^{-1}$ & 2 & 2,53 & 1,2 & 1,52 & 1 & 1,26 \\
\hline
\end{tabular}

Отраженные графиками в Рисунке 1 результаты вычислений выявляют согласие полученных результатов количественного моделирования исследуемого процесса с физическим 
смыслом задачи. А именно, с одной стороны, обессахаривание раствора по массе за счет кристаллизации сахарозы с течением времени растет (все кривые на Рисунке 1), такая же очевидная зависимость имеет место и при увеличении объемной концентрации порошка (Рисунок 1, например, кривая 2 выше кривой 1). Кроме того, в каждый момент времени и при одинаковой объемной концентрации по шарам разного размера, также, вследствие уменьшения удельной поверхности шара, для частиц большего радиуса, подача сахарозы к крупным шарам снижается (Рисунок 1, например, кривая 1 выше кривых 3 и 5).

Те же закономерности отмечаются и при визуализации данных рисунка 2. Например, время обработки раствора сокращается, когда концентрация затравочного материала увеличивается (кривая 2 ниже кривой 1). В свою очередь, вследствие уменьшения удельной поверхности шара для частиц большего радиуса, период времени проведения процесса обработки возрастает (кривая 3 выше кривой 1).

\section{Выводы}

Исходя из модели диффузионного массопереноса

$\mathrm{m}, \mathrm{kr}$

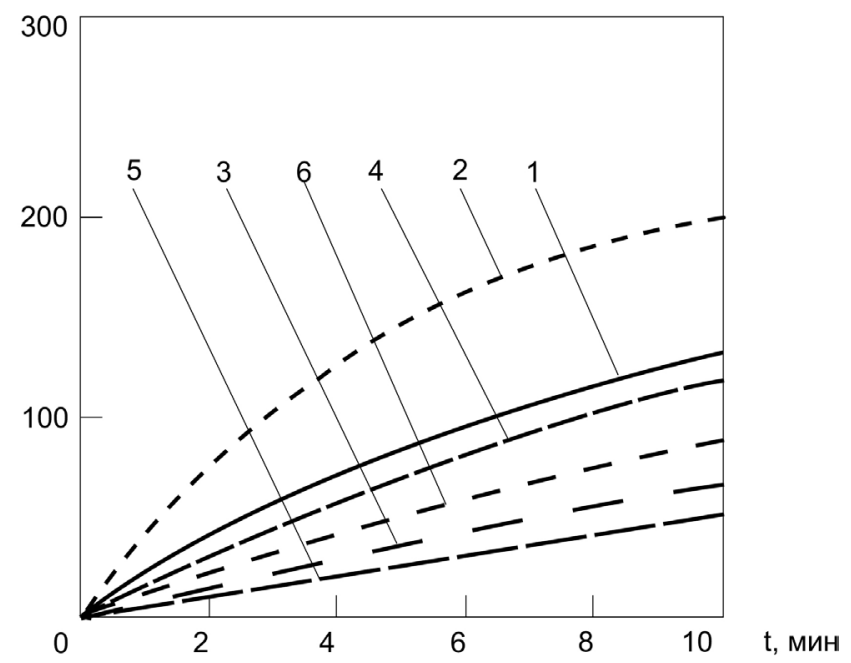

Рисунок 1. Зависимость массы кристаллической сахарозы (кг) в $1 \mathrm{~m}^{3}$ рабочего объема емкости от времени $t$ (мин) обработки продукта, для различных значений радиуса $R$ (м) частицы затравки и ее объемной концентрации $v(\%)$ в растворе $(\Delta \mathrm{c}=0,15$; $R=10^{-5} \mathrm{M}: 1-u=2 \times 10^{-4} \% ; 2-u=4 \times 10^{-4} \% ; R=2 \times 10^{-5}$ M: $5-u=2 \times 10^{-4} \% ; 6-u=4 \times 10^{-4} \%, R_{\mathrm{c}}=1,67 \times 10^{-5} \mathrm{M}$ : $\left.3-u=2 \times 10^{-4} \% ; 4-u=4 \times 10^{-4} \%\right)$. целевого продукта типа сахарозы от пересыщенного межкристального раствора к совокупности имитирующих затравку из тонкодисперсных полидисперсных частиц шарообразной формы, обосновывается физикоматематическая модель по прогнозированию процесса кристаллизации сахарозы в растворе.

Предлагается аналитический и расчетный аппарат по численному моделированию процесса кристаллизации целевого продукта типа сахарозы с точки зрения количества конденсированного на затравке вещества и времени проведения процесса обработки продукта.

\section{Литература}

Альтернативная технология кристаллизации сахарозы / Еделев Д.А. [и др.] //Хранение и переработка сельхозсырья. 2016. № 1. С. 5-11.

Будак Б.М., Самарский А.А., Тихонов А.Н. Сборник задач по математической физике. М.: ГИТТЛ, $1956.684 \mathrm{c}$.

Виноградова Ю.В., Гнездилова А.И. Влияние пересыщения, интенсивности перемешивания и температуры на кинетику массовой кристаллизации лактозы // сборник трудов конференции Вологодской государственной

$\tau$, мин

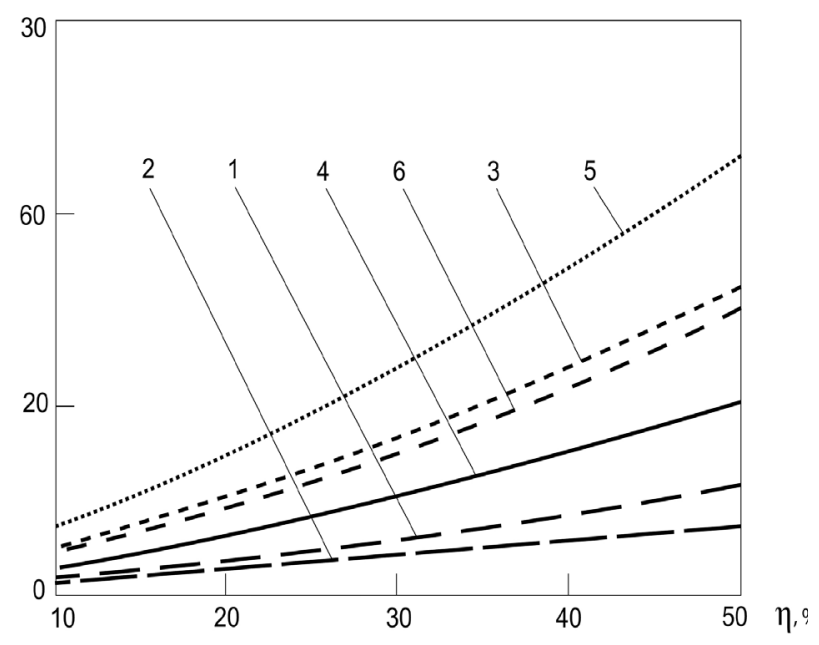

Рисунок 2. Зависимость времени $\tau$ (мин) от относительного (по предельному, \%) значению $\eta$ оседающего твердого $\left(\Delta \mathrm{c}=0,15 ; \mathrm{R}=10^{-5} \mathrm{M}: 1-\mathrm{u}=\right.$ $2 \times 10^{-4} \% ; 2-\mathrm{u}=4 \times 10^{-4} \% ; \mathrm{R}=2 \times 10^{-5} \mathrm{M}: 5-\mathrm{u}=2 \times 10^{-4}$ $\% ; 6-u=4 \times 10^{-4} \%$; Rc $=1,67 \times 10^{-5} \mathrm{M}: 3-\mathrm{u}=2 \times 10^{-4} \%$; $\left.4-u=4 \times 10^{-4} \%\right)$. 
молочно-хозяйственной академии им. Н.В. Верещагина. Вологда: Вологодская ГМХА, 2009. C. 18-24.

Гнездилова А.И., Шевчук В.Б. Кинетика массовой кристаллизации лактозы в пересыщенных водных растворах // сборник трудов конференции Вологодской государственной молочно-хозяйственной академии им. Н.В. Верещагина. Вологда: Вологодская ГМХА, 2005. C. 43-46.

Громковский А.И., Последова Ю.И., Бражников Н.Н. Оптимальный режим уваривания утфеля I продукта//Сахар. 2008. № 8. С. 54-56.

Комплексная оценка основных типов диффузионных установок свеклосахарного производства/ Валовой Б.Н. [и др.] // Сахар. 2016. № 11. С. 24-31.

Лебедева Н.Н. Разработка эффективной технологии уваривания утфеля первой кристаллизации: дис. на соиск. ученой степ. канд. техн. наук: 05.18.05: утв. 25.12.2013. М. 2013. 199 с.

Лыков А.В. Теория теплопроводности. М.: Высшая школа, 1967. 600 с.

Математическая модель оперативного учета и контроля свеклосахарного производства / Тужилкин В.И. [и др.] //Известия высших учебных заведений. Пищевая технология. 2018. №2-3(362-363). С. 117-121.

Михатова Г.Н., Каганов И.Н. Расчет продуктов сахарного производства. М.: Пищевая промышленность, 1973. 160 с.

Моделирование альтернативной технологии полунепрерывной кристаллизации сахарозы / Тужилкин В.И. [др.] //Известия высших учебных заведений. Пищевая технология. 2017. № 1 (355). С. $42-48$.

Моделирование уваривания утфелей с последовательной перетяжкой по ступеням кристаллизации в сравнении с традиционной технологией / Тужилкин В.И. [и др.] //Известия высших учебных заведений. Пищевая технология. 2015. №5-6 (347-348). С. 52-58.

Новые технологии кристаллизации сахарозы. / Тужилкин В.И. [ и др.] // Достижения науки и техники АПК, 2018. Т. 32, №12. С. 82-85.

Обоснование новых технологий изобарической и политермической кристаллизации сахарозы. / Тужилкин В.И. [и др.] // Инновационные решения при производстве продуктов питания из растительного сырья: материалы Международной научно-практической конференции. Воронеж: Издательскополиграфический центр «Научная книга», 2014.
C. $48-55$.

Особенности

диффузионного

процесса

кристаллизации сахарозы / Семенов Е.В. [и др.] //Сахар. 2013.№ 3. С. 46-50.

Повышение качества белого сахара при уваривании утфеля в горизонтальных вакуум-аппаратах непрерывного действия / Петров С.М. [и др.] // Caxap. 2018. № 6. C. 38-43.

Повышение качества свекловичного сахара до экспортного уровня / Петров С.М. [и др.]//Сахар. 2017. № 5. С. 30-33.

Полянский К.К., Шестов А.Г. Кристаллизация лактозы: физико-химические основы. Воронеж: ВГУ, 1995. 184 с.

Преимущества непрерывного уваривания утфеля / Петров С.М. [и др.] //Сахар. 2017. № 4. С. 30-37.

Сапронов А.Р. Технология сахарного производства. 2-е изд., испр. и доп. М.: Колос, 1999. 496 с.

Семенов Е.В., Славянский А.А., Ильина В.В. К расчету периода кристаллизации сахарозы в вакуум-аппарате//Хранение и переработка сельхозсырья. 2004. №12. С. 23-25.

Силин П.М. Технология сахара. 2-е изд., перераб. и доп. М.: Пищевая промышленность, 1967. 626 с.

Технологические и экономические аспекты кристаллизации сахара / Тужилкин В.И. [и др.] //Пищевая промышленность. 2014. №4. С. 66-69.

Фольмер М. Кинетика образования новой фазы. M.: Наука, 1986. 208 c.

Brown D.J., K.A. and F. Boysan. Crystal growth measurement and modeling of fluid flow in a crystallizer // Zuckerind. 1992. V. 117, № 1. P. 35-39.

Cecilia P.B., Librado G.R., Mayade G.O. Estudio de un agentetensoactivocubano y suefectosobre la velocidad de cristalización de la sacarose // Cent. Rev. Min. educ. super Rep. Cuba. 1984. V. 11, № 1. P. 121-132.

Grimsey I.M., Herrington T.M. The formation of inclusions in sucrose crystals // Int. Sugar J. 1994. V. 96, № 1152. P. 504-514.

Lin L., Siguan G., Bing L. Study on the hydrodynamic problems in the crystal growth from solution // J.S. China Univ. Technol. natur. Sci. 1996. V. 24, № 6. P. 25-29.

Mantovani G. Growth and morphology of sucrose crystal // Int. Sugar J. 1991. V. 93, № 1106. P. 23-32.

Pautrat C., Senotelle J., Matholouthi M. Effect of some polusaccharidicnon-sugar on the rate of sucrose crystal growth // Int. Sugar J. 1996. № 1171. P. 377384.

Pautrat C., Senotelle J., Matholoiithi M. Growth crystal sucrose at affect impurites // Int. SugarJ. 1996. № 137. P. 101-109. 


\title{
Peculiarities of the Process of Crystallization in Supersaturated Solution (On the Example of Sugar Production)
}

\author{
Anatoliy A. Slavyanskiy \\ Moscow State University of Technology and Management named after K.G. Razumovsky \\ (the First Cossacs University) \\ 73, Zemlyanoy Val str., Moscow, 109004, Russian Federation \\ E-mail: slavyanskiyaa@mgutm.ru \\ Evgeniy V. Semenov \\ Moscow State University of Technology and Management named after K.G. Razumovsky \\ (the First Cossacs University) \\ 73, Zemlyanoy Val str., Moscow, 109004, Russian Federation \\ 7E-mail:sem-post@mail.ru \\ Vera A. Gribkova \\ Moscow State University of Technology and Management named after K.G. Razumovsky \\ (the First Cossacs University) \\ 73, Zemlyanoy Val str., Moscow, 109004, Russian Federation \\ E-mail:vera_gribkova@list.ru \\ Natalia V. Nikolaeva
Moscow State University of Technology and Management named after K.G. Razumovsky
(the First Cossacs University)
73, Zemlyanoy Val str., Moscow, 109004, Russian Federation
E-mail: nata_nik@inbox.ru
}

The substance condensation processes on the seed material in a supersaturated solution are widespread in the processing industries of agrarian and industrial complex. So, this process, regarded as the phenomenon of the sucrose crystallization in the vacuum apparatus, is a hub in the technological flow of receiving crystal sugar. At the same time, prepared initial seed product in the form of finely powdered sucrose varies in shape and size, concentration in solution, etc. However, the theoretical basis of the phenomenon of solid-phase condensation on particles of seed material, as crystallization centrys, is not well developed to these factors. In the article, to validate a physical and mathematical model of the task, a spherical layer with a center in the ball modelling the sucrose particle is used as the volume of the solution surrounding the sucrose particle. In the analysis of the task come from the results of the problem of the heat transfer in the spherical layer associated with the theory of diffusive transfer of substance. The algorithm of quantitative research on the dependence of sucrose concentration on the parameters and the period of product processing is justified. A numerical experiment used as a means of quantitative analysis of the problem. This experiment revealed the adequacy of the results of theoretical analysis to the physical meaning of the actual process. Important theoretical and practical relationships have been obtained for estimating the mass of crystallized sucrose and the time of desugaring of the solution.

Keywords: solution of sucrose, the process of mass transfer, diffusion, period, crystallization, supersaturation, microterry solution

\section{References}

Budak B.M., Samarskiy A.A., Tikhonov A.N. Sbornik zadach po matematicheskoj fizike [Collection of problems in mathematical physics]. Moscow: GITTL, 1956. 684 p.Valovoj B.N., Filatov S.L., Petrov S.M., Podgornova N.M., Tugilkin V.I.

Kompleksnaj otsenka osnovnyk htipov diffuzionnykh ustanovok sveklosakharnogo proizvodstva [Comprehensive assessment of the main types of 
diffusion plants of beet sugar production]. Sakhar [Sugar], 2016, no. 11. pp. 24-31.

Vinogradova Y.V., Gnezdilova A.I. Vliyanie peresyshieniya, intensivnosti peremeshivaniya $\mathrm{i}$ temperatury na kinetiku massovoj kristallizatsii laktozy [Influence of supersaturation, mixing intensity and temperature on the kinetics of mass crystallization of lactose]. Sb. statejkonferentsii Vologodskoy gosudarstvennoy molochnohoziaystvennoy akademii im. N.V. Vereshchagina [proceedings of the conference of the Vologda state dairy Academy. im. N.V. Vereshchagin]. Vologda: 2009, pp. 18-24.

Gnezdilova A.I., Shevchuk V.B. Kinetika massovoy kristallizatsii laktozy $\mathrm{v}$ peresyshienykh vodnykh rastvorakh [Kinetics of mass crystallization of lactose in supersaturated aqueous solutions]. Sb. statejkonferentsii Vologodskoy gosudarstvennoy molochno-hoziaystvennoy akademiiim. N.V. Vereshchagina [proceedings of the conference of the Vologda state dairy Academy. im. N.V. Vereshchagin]. Vologda: 2005, pp. 43-46.

Gromovsriy A.I., Posledova Y.I., Brazhnikov N.N. Optimalinyi rezhim uvarivaniya utfelya I produkta [The optimal mode of boiling of the massecuite I product]. Sakhar [Sugar], 2008, no. 8. pp. 54-56.

Edelev D.A., Tuzhilkin V.I., Kovalenok V.A., Klemeshov D.A., Moyiseyak M.B., Filinov M.A., Urazbaeva K.A. Alternativnaya tekhnologiya kristallizatsii sakharozy [Alternative technology of crystallization of sucrose]. Hranenie i pererabotka sekkhozsyria [Storage and processing of agricultural raw materials], 2016, no 1, pp. 5-11.

Lebedeva N.N. Razrabotka effektivnoy tekhnologii uvarivaniya utfelya pervoy kristallizatsii. Diss. cand. tekhn. nauk. [The development of efficient technology for boiling of massecuite first crystallization. Dissertation of candidate of technical Sciences]. Moscow, 2013. 199 p.

Lykov A.V. Teoriya teploprovodnosti [Theory of thermal conductivity]. Moscow: Vyshsaya shkola, $1967,600 \mathrm{p}$.

Mikhatova G.N., Kaganov I.N. Raschet produktov sakharnogo proizvodstva [Calculation of sugar production products]. Moscow: Pishievaya promyshlennoct, 1973, $160 \mathrm{p}$.

Petrov S.M., Podgornova N.M., Tuzhilkin V.I., Filatov S.L. Povyshenie kachestvas veklovichnogo sakhara do eksportnogo urovnya [Improving the quality of beet sugar to the export level]. Sakhar [Sugar], 2017, no. 5. pp. 30-33.

Petrov S.M., Podgornova N.M., Tuzhilkin V.I., Filatov S.L. Preimushiestva nepreryvnogo uvarivaniya utfelya [Advantages of continuous boiling of massecuite]. Sakhar [Sugar], 2017, no. 4, pp. 30-37.

Petrov S.M., Filatov S.L. Podgornova N.M., Tuzhilkin
V.I. Povyshenie kachestva belogo sakhara pri uvarivanii utfelya $\mathrm{V}$ gorizontalnykh vacuum apparatakh nepreryvnogo deoystviya [Improving the quality of white sugar when boiling utfel in horizontal vacuum apparatus of continuous action]. Sakhar [Sugar], 2018, no. 6, pp. 38-43.

Polyanskiy K.K., Shestov A.G. Kristallizachiya laktozy: fiziko-khimicheskieosnovy [Crystallization of lactose: physical and chemical bases]. Voronezh: VGU, 1995. $184 \mathrm{p}$.

Sapronov A.P. Tekhnologiya sakharnogo proizvodstva [Sugar production technology]. 2 edition, revised and supplemented. Moscow: Kolos, 1999. 496 p.

Semenov E.V., Slavianskiy A.A., Ilina V.V. Raschet $\mathrm{u}$ perioda kristallizatsii sakharozy $\mathrm{V}$ vacuumapparate [To the calculation of the crystallization period of sucrose in a vacuum apparatus]. Hranenie i pererabotka sekkhozsyria [Storage and processing of agricultural raw materials], 2004, no. 12, pp. 2325.

Semenov E.V., Slavianskiy A.A., Sergeeva E.A., Shitova T.A. Osobtnnosti diffuzionnogo protsessa kristallizatsii sakharozy [Features of the diffusion process of sucrose crystallization]. Sakhar [Sugar], 2013, no. 3, pp. 46-50.

Silin P.M. Tekhnologiya sakhara [Technology of sugar]. 2 edition, revised and supplemented. Moscow: Pishievayapromyshlennoct, 1967, 626 p.

Tuzhilkin V.I., Balykhin M.G., Lukin N.D., Kovalenok V.A. Novye tekhnologii kristallizatsii sakharozy [The new technology of crystallization of sucrose]. Dostizhtniya nauki i tekhniki APK [Achievements of science and technology of agricultural and industrial complex], 2018, vol..32, no. 12, pp. 82-85.

Tuzhilkin V.I., Klemeshov D.A., Donenko G.A., Lukin N.D., Eshimbetova B.T. Matematicheskya model operativnogo ucheta i kontrolya sveklosakharnogo proizvodstva [Mathematical model of operational accounting and control of sugar beet production]. Izvestiya vysshikh uchtbnykhz avedeniy. Pishtvayatekhnologiya [Proceedings of higher educational institutions. Food technology], 2018, no. 2-3 (362-363), pp. 117-121.

Tuzhilkin V.I., Kovalenok V.A., Moyiseyak M.B., Urazbaeva K.A., Klemeshov D.A., Prokofiev E.N. Tekhnologicheskie i ekonomicheskie aspekty kristallizatsii sakhara [Technological and economic aspects of sugar crystallization]. Pishievaya promyshlennost [Food industry], 2014, no. 4, pp. 66-69.

Tuzhilkin V.I., Kovalenok V.A., Moyiseyak M.B., Klemeshov D.A., Achkasova N.Y., Urazbaeva K.A. Modelirovanie uvarivaniya utfeley $s$ posledovatelnoy peretyazhkoy postupenyam kristallizatsii $\mathrm{V}$ sravnenii $\mathrm{s}$ traditsionnoy tekhnologiey [Modeling of boiling of utpala with 
consistent padding on the degrees of crystallization in comparison with conventional technology]. Izvestiya vysshikh uchtbnykh zavedeniy. Pishevaya tekhnologiya [Proceedings of higher educational institutions. Food technology], 2015, no. 5-6 (347348), pp. 52-58.

Tuzhilkin V.I., Kovalenok V.A., Petrov S.M., Shterman V.S., Klemeshov D.A., Moyiseyak M.B., GavrilovA.M. Obosnovanie novykh tekhnologiy izobaricheskoy i politermicheskoy sakharozy [Justification of new technology and the Isobaric polythermal crystallization of sucrose]. Innovatsionnye resheniya pri proizvodstve produktov pitaniya izr astitelnogos yria: $v$ sbornike mezhdunarodnoj nauchno-prakticheskoj konferentsii [Innovative solutions in the production of food from vegetable raw materials: Proceedings of the 221st International scientific and practical conference]. Moscow, 2014, pp. 48-55.

Tuzhilkin V.I., Moyiseyak M.B., Klemeshov D.A., Kovalenok V.A. Modelirovanie alternativnoy tekhnologii polunepreryvnoy kristallizatsii sakharozi [Modeling of alternative technology of semi-continuous crystallization of sucrose]. Izvestiya vysshikh uchtbnykhz avedeniy. Pishtvayatekhnologiya [Proceedings of higher educational institutions. Food technology], 2017, no. 1 (355), pp. 42-48.
Folmer M. Kinetika obrazovaniya novoy fazy [Kinetics of new phase formation]. Moscow: Nauka, 1986. $208 \mathrm{p}$.

Brown D.J., K.A. and F. Boysan. Crystal growth measurement and modeling of fluid flow in a crystallizer.//Zuckerind. 1992. V. 117. № 1. P. 35-39.

Cecilia P.B., Librado G.R., Mayade G.O. Estudio de un agentetensoactivocubano y suefectosobre la velocidad de cristalización de la sacarose.//Cent. Rev. Min. educ. super Rep. Cuba. 1984. 11. № 1. P. 121-132.

Grimsey I.M., Herrington T.M. The formation of inclusions in sucrose crystals//Int. Sugar J. 1994. V. 96, № 1152. P. 504-514.

Lin L., Siguan G., Bing L. Study on the hydrodynamic problems in the crystal growth from solution//J.S. China Univ. Technol. natur. Sci. - 1996. V. 24. № 6. P. 25-29.

Mantovani G. Growth and morphology of sucrose crystal//Int. Sugar J. 1991. V. 93. № 1106. P. 23-32.

Pautrat C., Senotelle J., Matholouthi M. Effect of some polusaccharidicnon-sugar on the rate of sucrose crystal growth.//Int. Sugar J. 1996. №1171. P. 377384.

Pautrat C., Senotelle J., Matholoiithi M. Growth crystal sucrose at affect impurites.//Int. SugarJ. 1996. №137. P. 101-109. 